\title{
Galactic Cosmic Ray Acceleration with Steep Spectra
}

\author{
Rebecca Diesing ${ }^{a, *}$ and Damiano Caprioli ${ }^{a, b}$ \\ ${ }^{a}$ Department of Astronomy and Astrophysics, The University of Chicago, \\ 5640 S Ellis Ave, Chicago, IL 60637, USA \\ ${ }^{b}$ Enrico Fermi Institute, The University of Chicago, Chicago, IL 60637, USA \\ E-mail: rrdiesing@uchicago.edu, caprioli@uchicago.edu
}

Galactic cosmic rays (CRs) are accelerated by astrophysical shocks, primarily supernova remnants (SNRs), via diffusive shock acceleration (DSA), an efficient mechanism that predicts power-law energy distributions of CRs. However, observations of both nonthermal SNR emission and Galactic CRs imply CR spectra that are steeper than the standard DSA prediction, $\propto E^{-2}$. Recent kinetic hybrid simulations suggest that such steep spectra may be the result of a "postcursor", or drift of CRs and magnetic structures with respect to the thermal plasma behind the shock. Using a semi-analytic model of non-linear DSA, we generalize this result to a wide range of astrophysical shocks. By accounting for the presence of a postcursor, we produce CR energy distributions that are substantially steeper than $E^{-2}$ and consistent with observations. Our formalism reproduces both modestly steep spectra of Galactic SNRs $\left(\propto E^{-2.2}\right)$ and the very steep spectra of young radio supernovae $\left(\propto E^{-3}\right)$.

$37^{\text {th }}$ International Cosmic Ray Conference (ICRC 2021)

July 12 th - 23rd, 2021

Online - Berlin, Germany

\footnotetext{
*Presenter
} 


\section{Introduction}

Understanding the origin of Galactic cosmic rays (CRs) with energies up to $\sim 10^{8} \mathrm{GeV}$ requires a complete paradigm for their acceleration and propagation. The best source candidates for such acceleration are supernova remnants (SNRs), which provide sufficient energy and an efficient acceleration mechanism [13, 23, 49, 64]. In this mechanism, known as diffusive shock acceleration (DSA), particles are scattered by magnetic field perturbations, resulting in diffusion across the SNR forward shock and an energy gain with each crossing [6, 8, 15, 44, 54].

DSA predicts a power law momentum distribution of particles, $f_{\mathrm{sh}}(p) \propto p^{-q_{\mathrm{p}}}$, where $f_{\mathrm{sh}}(p)$ is the instantaneous momentum distribution of particles at the shock and $q_{\mathrm{p}}$ is set by the balance between the energy gained with each crossing and the escape of particles from the acceleration region [8]. Both of these quantities depend on the shock hydrodynamics such that $q_{\mathrm{p}}$ can be written in terms of the fluid compression ratio, $R=\rho_{2} / \rho_{0}$. Here, $\rho_{1}$ and $\rho_{2}$ are the densities of the fluid in front of the shock (upstream) and behind the shock (downstream) respectively. The relationship between $q_{\mathrm{p}}$ and $R$ reads, $q_{\mathrm{p}}=(3 R) /(R-1)$. For a strong shock with Mach number $M \gg 1, R=4$ and we obtain $q_{\mathrm{p}}=4$. Equivalently, DSA predicts power-law distributions in energy for relativistic particles, $\Phi_{\mathrm{sh}}(E) \propto E^{-q}$, where $\Phi_{\mathrm{sh}}(E)$ is the instantaneous energy distribution of particles at the shock. The relationship between $q$ and $R$ reads, $q=(R+2) /(R-1)$, with $q=2$ for a strong shock $(R=4)$.

A modification to the standard DSA prediction arises when CRs carry a non-negligible fraction of the shock's energy. When this occurs, CRs can no longer be treated as test-particles, resulting in modifications to the shock hydrodynamics and thus particle spectra [e.g., 11, 12, 14, 39, 40, $50,58,61,62]$. In this non-linear DSA (NLDSA), the CR pressure produces a region in front of the shock where the fluid is compressed, heated, and slowed. The presence of this region, or precursor, reduces the compression ratio near the shock into a subshock with $R_{\text {sub }} \equiv \rho_{2} / \rho_{1}<4$. Meanwhile, the total compression ratio between the downstream and far upstream becomes larger than the standard prediction: $R_{\text {tot }} \equiv \rho_{2} / \rho_{0}>4$. Note that, throughout this proceedings, subscripts $0,1,2$, and 3 are used to denote quantities at upstream infinity, immediately upstream of the shock, immediately downstream of the shock, and far downstream respectively.

As a result of these two compression ratios, NLDSA predicts concave CR spectra. More specifically, particles with lower energies remain close to the shock and probe $R_{\text {sub }}<4$, while particles with higher energies diffuse further upstream and probe $R_{\text {tot }}>4$. Thus, low/high energy particles are expected to exhibit spectra steeper/flatter than $E^{-2}$. The transition between these regimes occurs at the lowest energy where CRs carry non-negligible pressure, which is usually transrelativistic. Since the nonthermal emission in astrophysical environments is typically generated by relativistic CRs, the classical NLDSA theory predicts that observations of non-thermal emission from shock-powered sources should be explained by CR spectra flatter than $E^{-2}$.

\subsection{Theory vs. Observations}

This prediction is readily testable via observations of the nonthermal emission, e.g., from the relics of stellar explosions. However, the first GeV observations of SNRs, combined with preexisting $\mathrm{TeV}$ data, did not confirm the existence of concave spectra. On the contrary, they pointed toward 
CR acceleration with spectra steeper than $E^{-2}$ [21]. Notable examples include historical remnants such as Tycho's SNR $[q=2.3 \pm 0.2,5,45]$ and Cassiopeia A $[q=2.36 \pm 0.02$ above $17 \mathrm{GeV}, 66]$.

Further evidence for steep spectra comes in the form of SNR radio emission, particularly that of young, extragalactic supernovae (radio $\mathrm{SNe}$ ). These remnants exhibit synchrotron spectra that imply electron distributions as steep as $E^{-3}$ [e.g., 33, 34, 51, 71, 72]. However, it is possible that these synchrotron spectra probe the steep portion of a concave spectrum, since the electrons responsible are likely sub-GeV [e.g., 40, 41, 73].

The CR spectrum measured at Earth also points toward CR acceleration with spectra steeper than $E^{-2}$. In the standard picture of CR transport, this measured CR spectrum goes as $E^{-(q+\delta)}$, where $\delta$ is the slope of the CR residence time in the Galaxy: $\tau_{\text {res }} \propto E^{-\delta}$. Measurements of the $\mathrm{CR}$ anisotropy suggest $\delta \sim 0.3[16,17]$. Meanwhile, secondary to primary ratios suggest that $0.3 \lesssim \delta \lesssim 0.4$, depending on the CR energy [e.g., 2]. Thus, fitting the observed Galactic CR spectrum-which goes as $E^{-2.7}$-requires $2.3 \lesssim q \lesssim 2.4[42,43]$.

\subsection{A Revised Theory of DSA}

A number of explanations for steep CR spectra have been proposed in the literature. For a detailed summary of these explanations and their limitations, see [27].

One possible explanation considers the role of the magnetic fluctuations responsible for $\mathrm{CR}$ scattering [e.g., 21, 22, 53, 80]. In the standard DSA theory, particles are isotropized in both the upstream and downstream such that they "feel" a head-on collision with each crossing of the shock. The resulting energy gain per crossing thus depends on the difference in velocity between the upstream and downstream plasma, $u_{1}-u_{2}$. In reality, however, magnetic fluctuations-not thermal plasma-are responsible for particle scattering, meaning that particles will be isotropized in the fluctuation frame. The relative drift between the fluid and the fluctuations was already present in the early DSA theory [8], but it has been usually neglected because the fluctuation drift is roughly the Alfvén speed, much smaller than the fluid speed in the shock frame. In the presence of CRdriven magnetic field amplification, however, such a drift may be significantly enhanced; one can argue that, in the upstream, these fluctuations move against the fluid with the local Alfvén velocity in the amplified field, $v_{\mathrm{A}, 1}$ [e.g., 22]. Thus, CRs experience a smaller energy gain per crossing $\propto u_{1}-v_{\mathrm{A}, 1}-u_{2}$ or, equivalently, they "feel" a compression ratio, $\tilde{R}$ that is smaller than that of fluid,

$$
\tilde{R}=\frac{u_{1}-v_{\mathrm{A}, 1}}{u_{2}}<R=\frac{u_{1}}{u_{2}} .
$$

This prescription may naturally lead to spectra that are steeper than $E^{-2}$ [21, 22], and it has been used, e.g., to model for the broadband emission of Tycho's SNR $[60,70]$ and of intracluster shocks [52].

The potential role of such drifts had not been validated by self-consistent kinetic simulations until very recently, when [48] and [27] put forward unprecedentedly-long hybrid simulations (i.e., particle-in-cell simulations with kinetic ions and fluid electrons) that showed the onset of CRmodified shocks. That being said, the presence of a precursor is insufficient to explain the very steep spectra $\left(\propto E^{-3}\right)$ of radio $\mathrm{SNe}$, and its effect may be limited if magnetic field amplification in the upstream is spatially-dependent. In particular, if the local Alfvén speed decreases in the 
precursor, particles with long diffusion lengths will probe a region with reduced fluctuation drift, resulting in a flattening of the CR spectrum at the highest energies.

However, [48] finds that not only does a precursor form in front of the shock, in which selfgenerated fluctuations move at roughly $v_{\mathrm{A}, 1}$ in the amplified field, but also that the motion of magnetic structures behind the shock leads to the formation of a postcursor. In this picture, CRdriven magnetic fluctuations generated in the upstream retain their inertia over a non-negligible distance (larger than the CR diffusion length) when advected and compressed into the downstream. As a result, these fluctuations move away from the shock faster than the background plasma, or more specifically, with velocity $\tilde{u}_{2}=u_{2}+v_{\mathrm{A}, 2}$ with respect to the shock.

Since CRs tend to isotropize with magnetic fluctuations, they too experience a net drift equal to $v_{\mathrm{A}, 2}$ relative to the background plasma [see Figure 6 in 48]. These drifts away from the shock lead to the removal of CR and magnetic energy from the shock and thus an enhancement of the fluid compression ratio and a steepening of the CR spectrum, as discussed in Section 5 of [48].

Equivalently, one can think of the postcursor as modifying the compression ratio "felt" by CRs, just as the precursor modifies this ratio in [22]. In the postcursor paradigm (ignoring, for now, the presence of a precursor), we have,

$$
\tilde{R}=\frac{u_{1}}{u_{2}+v_{\mathrm{A}, 2}}=\frac{R}{1+\alpha},
$$

where $\alpha \equiv v_{\mathrm{A}, 2} / u_{2}$. Thus, $q_{\mathrm{p}}$ depends only on $R$ and $\alpha$ or, equivalently, on $R$ and the magnetic pressure fraction downstream, $\xi_{\mathrm{B}, 2} \equiv B_{2}^{2} /\left(8 \pi \rho_{0} v_{\mathrm{sh}}^{2}\right)$ :

$$
q_{\mathrm{p}}=\frac{3 R}{R-1-\alpha}=\frac{3 R}{R-1-\sqrt{2 R \xi_{\mathrm{B}, 2}}} .
$$

Note that the effect of the postcursor will dominate that of a precursor, since compression of the magnetic field in the downstream leads to $\alpha>v_{\mathrm{A}, 1} / u_{1}$ [27]. In the case of efficient CR acceleration and thus magnetic field amplification, [48] reports $\alpha \sim 0.6$, which is sufficient to produce spectra steeper than $p^{-4}$, or $E^{-2}$ at relativistic energies.

While these hybrid simulations provide a motivation and a physical explanation for the modification of the standard DSA theory, quantifying the steepening of the CR spectra in astrophysical systems requires additional calculations. Namely, the postcursor paradigm implies that spectral steepening increases with the downstream magnetic field strength, which, due to magnetic field amplification via CR-driven instabilities, increases with the CR pressure [e.g., 9, 35]. However, if spectra become too steep, the CR pressure will drop, reducing magnetic field amplification and thus causing the steepening to saturate.

In this proceedings, we use a semi-analytic model of NLDSA to generalize the results of [27] and estimate $q$ for a wide range of SNR shocks. The results presented in this proceedings can also be found in [38].

\section{Method}

To fully understand how a postcursor affects $\mathrm{CR}$ acceleration, we use a semi-analytic formalism to model SNR shocks over a range of ambient number densities, $n_{\mathrm{ISM}}$, ambient magnetic fields, $B_{0}$, 
and SN energies, $E_{\mathrm{SN}}$. Herein we describe this formalism briefly, including our models for SNR evolution, particle acceleration, and magnetic field amplification. A more detailed description of our model, particularly our prescription for particle acceleration, can be found in [22] and [37].

\subsection{Shock Hydrodynamics}

We model SNR shock hydrodynamics using the formalism described in [36], which includes the effect of CR pressure on the evolution of the shock. More specifically, SNR evolution is modeled through three stages spanning $\gtrsim 10^{5} \mathrm{yr}$ : the ejecta-dominated stage, in which the mass of the swept-up ambient gas is less than that of the SN ejecta, the Sedov stage, in which the sweptup mass dominates the total mass and the SNR expands adiabatically, and the pressure-driven snowplow, in which the remnant cools due to forbidden atomic transitions but continues to expand because its internal pressure exceeds the ambient pressure. After this point, the remnant enters the momentum-driven snowplow, in which the internal pressure falls below the ambient pressure and expansion continues due to momentum conservation.

All SNRs are assumed to eject $M_{\mathrm{ej}}=1 M_{\odot}(1$ solar mass $)$ with $E_{\mathrm{SN}} \in\left[10^{51}, 10^{52}\right]$ erg into a uniform ambient medium of density $n_{\mathrm{ISM}} \in\left[10^{-1}, 10^{5}\right] \mathrm{cm}^{-3}$ and magnetic field $B_{0} \in[3,3000] \mu \mathrm{G}$.

\subsection{Particle Acceleration}

We model CR acceleration using a semi-analytic model of NLDSA described in [22, 24, 26, 37] and references therein. This model self-consistently solves the diffusion-advection equation for the transport of non-thermal particles in a quasi-parallel, non-relativistic shock, including the dynamical backreaction of accelerated particles and of CR-generated magnetic turbulence.

Particles above a threshold in momentum, $p_{\text {inj }}$, are injected into the acceleration process, with $p_{\text {inj }} \equiv \xi_{\text {inj }} m_{\mathrm{p}} v_{\text {sh }} /\left(1+R_{\text {tot }}^{-1}\right)$, consistent with the parameterization described in [28], since $v_{\mathrm{sh}} /\left(1+R_{\mathrm{tot}}^{-1}\right)$ is simply the velocity of the upstream fluid in the downstream frame. In general, an increase in $\xi_{\text {inj }}$ corresponds to a decrease in the fraction of particles crossing the shock that are injected into DSA. Here we neglect the dependence of injection on the shock inclination and set an effective value of $\xi_{\text {inj }}=3.8$, which yields $\xi_{\mathrm{CR}} \equiv P_{\mathrm{CR}} /\left(\rho_{0} v_{\mathrm{sh}}^{2}\right) \approx 0.1$ for a prototypical SNR $\left(n_{\mathrm{ISM}}=1 \mathrm{~cm}^{-3}, B_{0}=3 \mu \mathrm{G}, E_{\mathrm{SN}}=10^{51} \mathrm{erg}, M_{\mathrm{ej}}=1 M_{\odot}\right)$ after a few hundred years, consistent with SNR observations. Note that $P_{\mathrm{CR}}$ refers to the CR pressure.

To account for the effects of a precursor and postcursor, we introduce into the diffusionadvection equation $\tilde{u}(x) \equiv u(x) \pm v_{\mathrm{A}}(x)$, the effective fluid velocity as felt by the non-thermal particles which are scattered by magnetic structures moving at $v_{\mathrm{A}}(x)$ relative to the thermal plasma. Note that these structures move against the fluid in the upstream, but with the fluid in the downstream [27]. Throughout this work, we assume that the postcursor extends beyond the diffusion length of the highest energy particles, i.e., behind the shock, $\tilde{u}(x)=u_{2}+v_{\mathrm{A}, 2}$.

The actual extent of the postcursor in astrophysical shocks is difficult to quantify, even if highresolution X-ray observations of individual SNRs with Chandra suggest that the magnetic field remains amplified on a scale of $1-5 \%$ of the SNR radius [e.g., 74]. Physically speaking, since the maximum CR energy $E_{\max }$ is controlled by the smallest between the upstream and the downstream diffusion length [e.g., 18, 56, 61], the post-shock region with high magnetic field must be at least as extended as the diffusion length of particles with $E_{\max }$. It follows that the postcursor must be more 
extended than the diffusion length of any particle, thereby leading to a global steepening of the CR spectrum. Note that, when only the Alfvénic drift in the precursor is retained [á la 22, 80], a global steepening is only possible if escaping CRs drive magnetic field amplification on all scales, which is not guaranteed.

In practice, our formalism begins with an initial guess for the CR pressure, which is used to solve the equations for conservation of mass, momentum, and energy across a plane, nonrelativistic shock. The magnetic field pressure, $P_{\mathrm{B}}$, is then calculated using the prescription described in 2.3, and the resulting $u(x)$ and $P_{\mathrm{B}}$ are then used to solve the diffusion-advection equation, which can be integrated to find a new guess for $P_{\mathrm{CR}}$. In this manner, our formalism iteratively solves for the $\mathrm{CR}$ spectrum while self-consistently accounting for the dynamical effect of accelerated particles and the amplification of magnetic fields.

Once the proton spectrum has been calculated at each timestep of SNR evolution, particle momenta are shifted and the instantaneous spectra are weighted to account for adiabatic losses [see 23, 37, 60, for more details]. These weighted contributions are then added together to obtain a cumulative spectrum.

\subsection{Magnetic Field Amplification}

The propagation of energetic particles ahead of the shock is expected to excite streaming instabilities, [4, 8, 9, 20], which drive magnetic field amplification and enhance CR diffusion $[29,30]$. The result is magnetic field perturbations with magnitudes that can exceed that of the ordered background magnetic field. This magnetic field amplification has been observationally inferred from the X-ray emission of many young SNRs, which exhibit narrow X-ray rims due to synchrotron losses by relativistic electrons [e.g., 7, 59, 63, 65].

We model magnetic field amplification by assuming contributions from both the resonant streaming instability [e.g., 55, 67-69, 81], and the non-resonant hybrid instability [9]. A detailed discussion of these instabilities and their saturation points can be found in [35].

In the resonant instability, CRs excite Alfvén waves with a wavelength matching their gyroradius. The growth of this instability saturates when the strength of magnetic perturbations reaches the level of the ordered background field: $\delta B / B \sim 1$. More specifically, [3] derives this saturation level to be $P_{\mathrm{B} 1 \text {,res }}=\left(P_{\mathrm{CR}, 1}\right) /\left(4 M_{\mathrm{A}, 0}\right)$, where $M_{\mathrm{A}} \equiv v_{\mathrm{sh}} / v_{\mathrm{A}, 0}$ is the Alfvénic Mach number.

For fast shocks typical of young SNRs, more significant is the non-resonant hybrid instability. Driven by CR currents, $\mathbf{j}$, in the upstream, [9] predicts that saturation occurs when tension in magnetic field lines becomes sufficient to oppose the $\mathbf{j} \times \mathbf{B}$ force or, equivalently, when the magnetic field pressure reaches approximate equipartition with the anisotropic fraction of the CR pressure [also see 19],

$$
P_{\mathrm{B} 1, \mathrm{Bell}}=\frac{v_{\mathrm{Sh}}}{2 c} \frac{P_{\mathrm{CR}, 1}}{\gamma_{\mathrm{CR}}-1} .
$$

Here, $c$ is the speed of light and $\gamma_{\mathrm{CR}}=4 / 3$ is the $\mathrm{CR}$ adiabatic index. This saturation can lead to $\delta B / B_{0} \gg 1$ and has been validated with hybrid simulations in [78].

To account for both the resonant and non-resonant instabilities, we pose here that the upstream magnetic field pressure is given by $P_{\mathrm{B}, 1}=\sqrt{P_{\mathrm{B} 1 \text {,res }}^{2}+P_{\mathrm{B} 1 \text {, Bell }}^{2}}$. Assuming that all components of the magnetic perturbations upstream are compressed, the downstream magnetic field strength is 


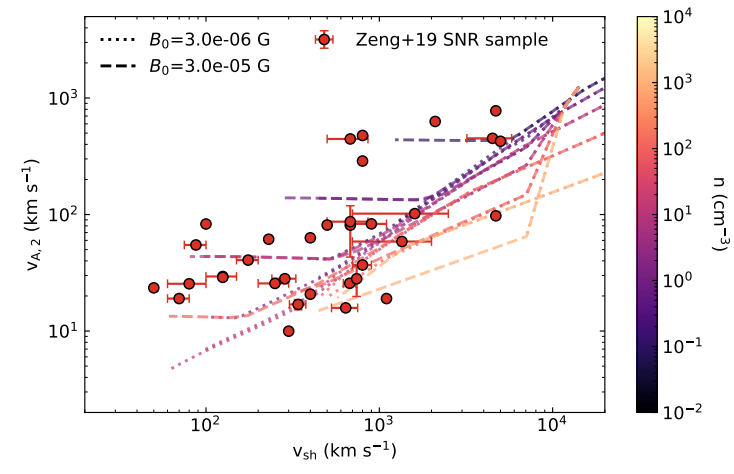

Figure 1: Downstream Alfvén speed, $v_{\mathrm{A}, 2}$, as a function of shock velocity, $v_{\mathrm{sh}}$ for a number of modeled SNR evolutions (dotted and dashed lines). Each line corresponds to a single evolution with a fixed ambient density (color scale) and ambient magnetic field (line style). Overlaid are the SNR data aggregated in [79]. Our prescription for magnetic field amplification produces modeled SNRs in good agreement with the measured relationship between $v_{\mathrm{A}, 2}$ and $v_{\mathrm{sh}}$.

$B_{2} \simeq R_{\text {sub }} B_{1}$. For an acceleration efficiency $\xi_{\mathrm{CR}} \approx 0.1$, our typical SNR parameters give $B_{2}$ near a few hundred $\mu \mathrm{G}$, in good agreement with X-ray observations of young SNRs [25, 63, 77].

For a more robust test of our prescription, we consider the relationship between $v_{\mathrm{sh}}$ and $v_{\mathrm{A}, 2}$. Specifically, our prescription predicts a positive relationship between $v_{\mathrm{sh}}$ and $v_{\mathrm{A}, 2}$ for large $v_{\mathrm{sh}}$ (i.e., where the non-resonant instability dominates). At lower $v_{\text {sh }}$ (i.e., where the resonant instability dominates), we would expect little to no correlation, since the resonant instability has a weaker dependence on $v_{\mathrm{sh}}$ and depends on the ambient magnetic field, which may vary. In Figure 1, we compare our predicted relationship between $v_{\mathrm{A}, 2}$ and $v_{\mathrm{sh}}$ to observational results compiled in [79]. As Figure 1 shows, our prescription yields a good agreement with observations. This agreement also provides circumstantial evidence that the presence of a postcursor is responsible for steep SNR spectra, particularly in light of the fact that SNRs with large $v_{\text {sh }}$ tend to have larger $q$ [e.g., 10].

\section{Results}

Herein we present our modeled CR spectra and quantify the steepening resulting from the modified shock dynamics-namely, the presence of a postcursor-described in [48] and [27]. We also compare our results to observations. Throughout this section, we estimate power-law slopes as $q \equiv-\langle d \log \Phi(E) / d \log E\rangle$, where $\Phi(E)=d N(E) / d E$ is the cumulative proton spectrum and $q$ is averaged between $10-10^{3} \mathrm{GeV}$.

\subsection{Spectral Steepening}

Our modeled spectrum of a "prototypical," or Tycho-like SNR $\left(n_{\mathrm{ISM}}=1 \mathrm{~cm}^{-3}, B_{0}=3 \mu \mathrm{G}\right.$, $E_{\mathrm{SN}}=10^{51} \mathrm{erg}$, and $M_{\mathrm{ej}}=1 M_{\odot}$ ) is shown in Figure 2a, including the contributions of protons accelerated at various stages of its evolution. These contributions are all steeper than $E^{-2}$, resulting in a cumulative spectrum $\Phi(E) \propto E^{-2.23}$ by the end of the SNR lifetime $\left(\sim 10^{5} \mathrm{yr}\right)$.

A more explicit quantification of the effect of the postcursor can be found in Figure 2b. Here, we compare the cumulative spectrum of our Tycho-like SNR after $400 \mathrm{yr}$ to the traditional NLDSA result and to the case with a postcursor but no net motion of magnetic structures in the precursor. As expected, the NLDSA formalism produces a modestly concave spectrum that deviates slightly from the standard $E^{-2}$ prediction. Meanwhile, the addition of a postcursor softens this spectrum substantially to $E^{-2.30}$. The addition of a precursor yields a slight increase in this steepening to $E^{-2.34}$, but its effect is underdominant due to the fact that the upstream magnetic field is decompressed such that $v_{\mathrm{A}, 1} / u_{1}<v_{\mathrm{A}, 2} / u_{2}$. 

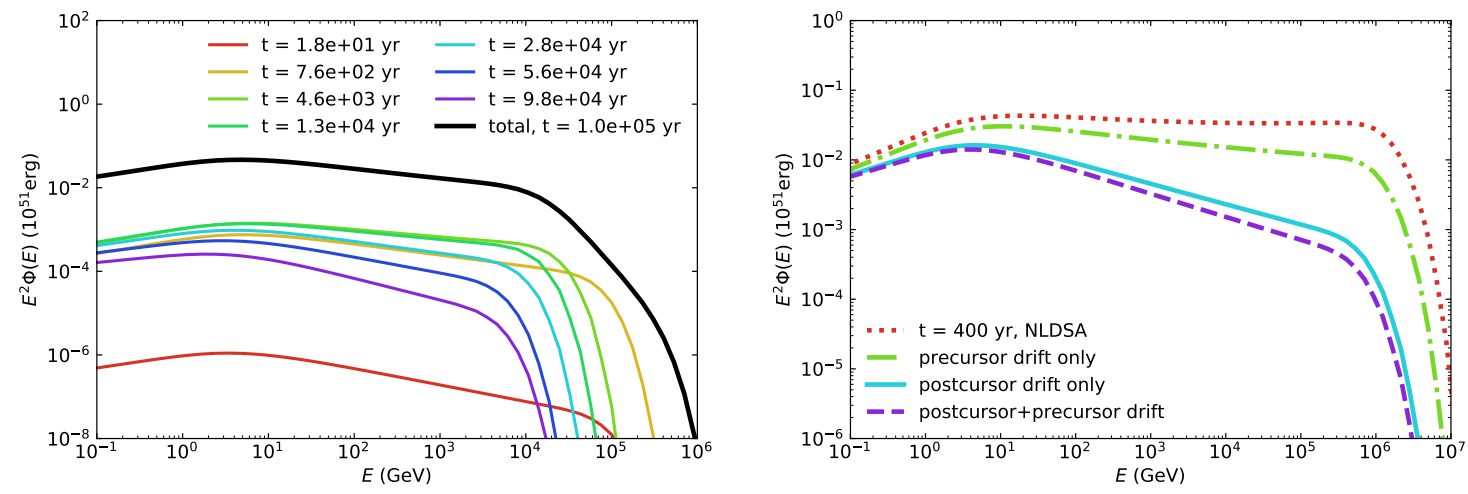

Figure 2: Left: The modeled proton distribution, $\Phi(E)$, for a Tycho-like SNR with precursor and postcursor drift included: $n_{\mathrm{ISM}}=1 \mathrm{~cm}^{-3}, B_{0}=3 \mu \mathrm{G}, E_{\mathrm{SN}}=10^{51} \mathrm{erg}$, and $M_{\mathrm{ej}}=1 M_{\odot}$. The black line shows the cumulative proton spectrum after $10^{5} \mathrm{yr}$, while the colored lines show the contributions to this final spectrum from various timesteps. Throughout the SNR's evolution, protons are accelerated with spectra steeper than $E^{-2}$. Right: The modeled proton distribution of the same Tycho-like SNR after 400 yr. Spectra are shown assuming traditional NLDSA with no net drift of magnetic fluctuations (red dotted line), assuming net drift in the precursor only (green dot-dashed line), assuming net drift in the postcursor only (blue solid line), and assuming the net drift in both the precursor and postcursor (purple dashed line). The inclusion of postcursor drift produces a substantial spectral steepening relative to the traditional NLDSA prediction. The addition of precursor drift further steepens the proton spectrum, but its effect is subdominant.

A summary of our results can be found in Figure 5, which shows the average power law slope, $q$, as a function of shock velocity, $v_{\mathrm{sh}}$, for the full range of modeled SNRs described in 2.1. To span a larger velocity range, we include models with initial energy, $E_{\mathrm{SN}}$, between $10^{51}$ and $10^{52} \mathrm{erg}$. Since increasing $E_{\mathrm{SN}}$ increases the shock velocity but does not otherwise affect shock hydrodynamics, we do not visually distinguish between different $E_{\mathrm{SN}}$ in Figure 5 . A fast shock may therefore correspond to a large $E_{\mathrm{SN}}$ or a young SNR; from the perspective of CR acceleration and magnetic field amplification, the two scenarios are equivalent. For this reason, our parameter range effectively spans different ejecta masses as well. Namely, an increase in $M_{\mathrm{ej}}$ simply corresponds to a decrease in $v_{\mathrm{Sh}}$ for a given $E_{\mathrm{SN}}$. With the range of SNR parameters described in 2.1, we obtain $2.1 \lesssim q \lesssim 3$.

For large $v_{\text {sh }}$ an increase in $v_{\text {sh }}$ corresponds to an increase in $q$, as one would expect when the Bell instability drives magnetic field amplification. As suggested in Figure 2a, this dependence disappears when $v_{\text {sh }}$ becomes small enough that the resonant instability dominates. The velocity where this transition occurs depends on the ambient density and magnetic field, introducing a spread in the relationship between $q$ and $v_{\mathrm{sh}}$ which, in the case of small ambient densities and large magnetic fields, can extend up to high $v_{\mathrm{sh}}\left(\gtrsim 10^{4} \mathrm{~km} \mathrm{~s}^{-1}\right)$.

\subsection{Comparison to Observations}

By solving the equations for conservation of mass, momentum, and energy across a postcursormodified shock, one can predict the fluid compression ratio as a function of the $\mathrm{CR}$ acceleration efficiency, $\xi_{\mathrm{CR}}$, and the magnetic pressure fraction, $\xi_{\mathrm{B}}$. Thus, the postcursor paradigm predicts a 

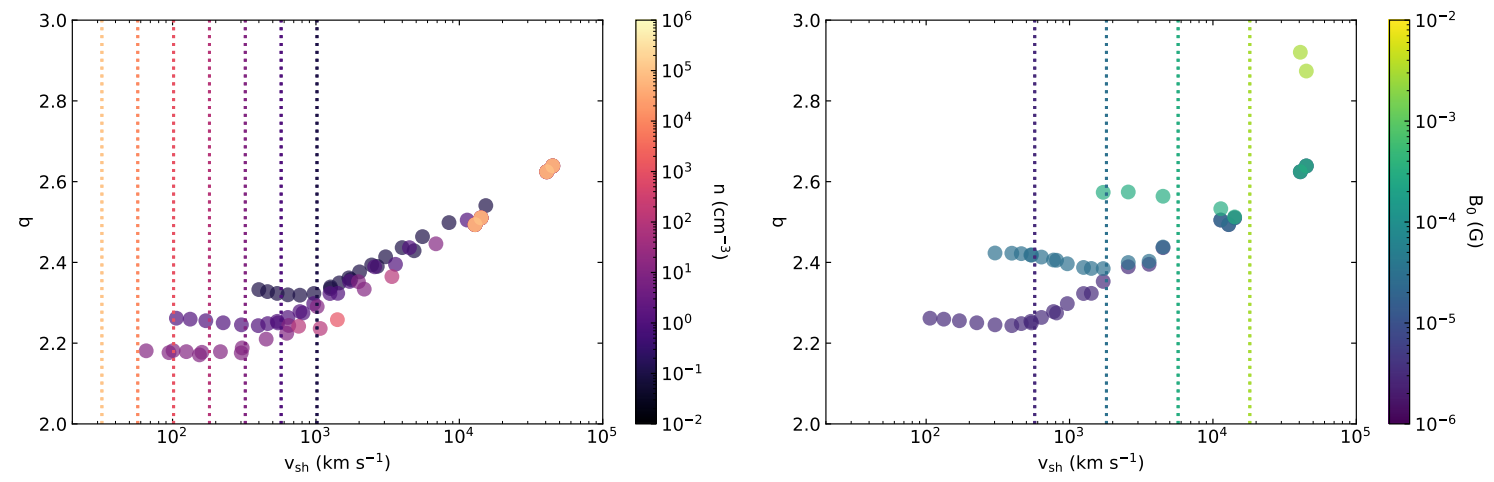

Figure 3: Power-law slopes, $q$, of modeled proton spectra as a function of shock velocity. The dotted vertical lines correspond to $v_{\mathrm{sh}}^{*}$, the shock velocity where, for a given ambient density or magnetic field denoted by the color scale, the dominant source of magnetic field amplification transitions from the resonant to the non-resonant instability. Left: The ambient magnetic field is held fixed at $3 \mu \mathrm{G}$ while density, denoted by the color scale, is varied. Right: The ambient density is held fixed at $1 \mathrm{~cm}^{-3}$ while ambient magnetic field, again denoted by the color scale, is varied. In general, faster shocks give rise to larger magnetic field amplification and thus steeper spectra. However, this dependence on shock velocity disappears at low velocities where the resonant streaming instability is the primary source of magnetic field amplification.

well-defined relationship between $q, \xi_{\mathrm{CR}}$, and $\xi_{\mathrm{B}}$. Assuming magnetic field amplification is driven by the non-resonant instability, $\xi_{\mathrm{B}}$ can be recast in terms of $\xi_{\mathrm{CR}}$ and $v_{\mathrm{sh}}$, meaning that observational constraints on the shock velocity and spectral slope correspond to constraints on the CR acceleration efficiency. For reference, we summarize this relationship in Figure 4, assuming CRs probe the full compression ratio from the far upstream to the downstream.

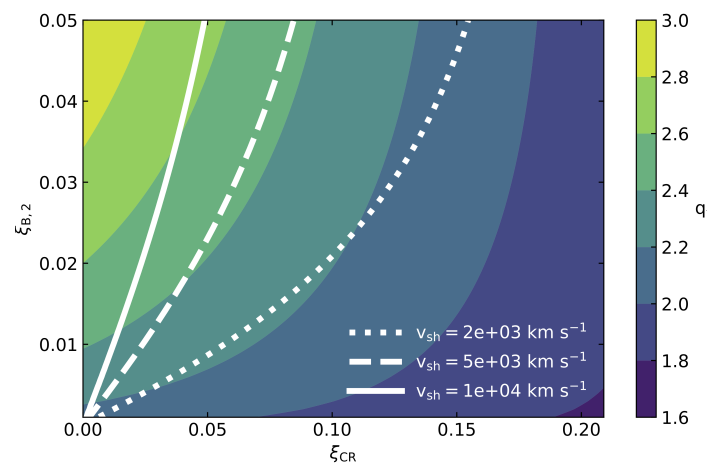

Figure 4: Predicted power law slope, $q$, denoted by color scale, as a function of CR acceleration efficiency, $\xi_{\mathrm{CR}}$, and magnetic pressure fraction downstream, $\xi_{\mathrm{B}, 2}$. $q$ is calculated for a strong shock assuming CRs probe the full compression ratio from the far upstream to the downstream (regions 0 and 2 respectively. White lines denoting $\xi_{\mathrm{B}, 2}$ as a function of $\xi_{\mathrm{CR}}$ for various shock velocities are overlaid, assuming magnetic field amplification is dominated by the non-resonant instability.

Equivalently, we can test the validity of the postcursor paradigm by comparing our predicted spectra to to observations, in particular the non-thermal emission of Galactic remnants (including historical SNRs), and young extragalactic supernovae (radio SNe). The SNRs in our Galaxy consist largely of older, slower shocks $\left[v_{\mathrm{sh}} \ll 10^{4} \mathrm{~km} \mathrm{~s}^{-1}\right.$, see, e.g., 46]. Assuming magnetic field amplification driven by the non-resonant instability, we would therefore expect these SNRsin the postcursor paradigm-to exhibit only modestly steep spectra. To test this, we look to GeV observations aggregated in [21] from the Fermi LAT source catalog [1]. To ensure accurate estimates of $q$, we remove SNRs with GeV emission that is likely leptonic in origin: RX J1713.7-3946 [e.g., 

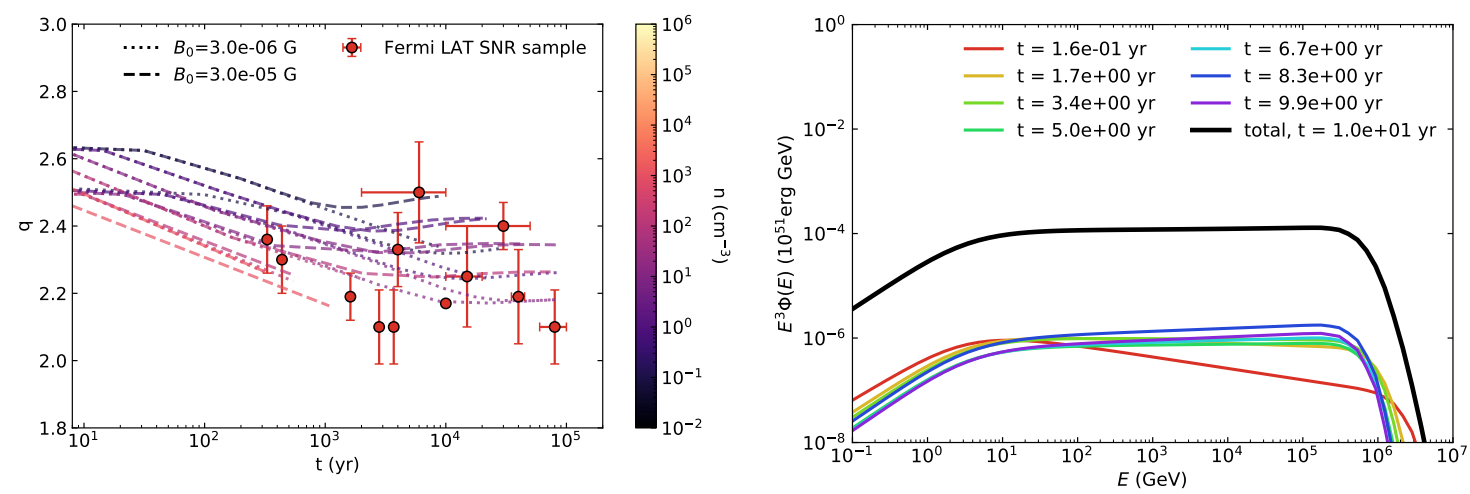

Figure 5: Left: Power law slopes, $q$, of modeled proton spectra (dotted an dashed lines) as a function of SNR age. The slopes of GeV spectra from the Fermi LAT catalog [1] are overlaid. For simplicity, SNRs have been removed if their $\mathrm{GeV}$ emission is likely leptonic in origin or exhibits a significant spectral break (see text for details). The spectral information for Cassiopeia A has been taken from [66]. The inclusion of a postcursor produces steep proton spectra in good agreement with SNR observations. Right: The modeled proton distribution $\left(E^{3} \Phi(E)\right)$ for a sample radio SN expanding into a circumstellar wind ( $n_{\text {ISM }} \propto r^{-2}$; see text for details). The black line shows the cumulative proton spectrum after $10 \mathrm{yr}$, while the colored lines show the contributions to this spectrum from various timesteps. Our toy model reproduces the very steep spectra characteristic of radio $\mathrm{SNe}$; for this setup, we obtain $q \simeq 2.99$.

47] and Vela Jr. [e.g., 57]. For simplicity, we also remove SNRs with breaks or cutoffs in the $\mathrm{GeV}$ range, which are typically interpreted as due to reacceleration [e.g., W44, see 31]. When possible, we use results from combined $\mathrm{GeV}-\mathrm{TeV}$ analyses [e.g., for Cas A, 66], which provide a more accurate representation of the full $\gamma$-ray slope.

Figure 5a compares the values of $q$ calculated for our modeled SNRs to those in our sample. Our models are able to reproduce the full range of slopes inferred from $\mathrm{GeV}$ and $\mathrm{TeV}$ observations of Galactic SNRs: $2.1 \lesssim q \lesssim 2.6$.

In addition to explaining the modestly steep spectra of Galactic SNRs, the presence of a postcursor may also explain the very steep spectra of their extragalactic counterparts: radio SNe. These young, fast remnants $\left(v_{\mathrm{sh}} \gtrsim 10^{4} \mathrm{~km} \mathrm{~s}^{-1}\right)$ typically expand into dense circumstellar winds blown by the progenitor star [see, e.g., 34]. Their high $v_{\text {sh }}$ and, more explicitly, their large inferred postshock magnetic fields $[\sim 0.1-1 \mathrm{G}$, see, e.g., 32] imply strong magnetic field amplification, making them excellent candidates for tests of postcursor physics.

Intriguingly, radio SNe exhibit synchrotron emission that suggest electron distributions $\Phi(E) \propto$ $E^{-3}$ or even steeper [see, e.g., 33, 51, 71, 72]. Assuming protons and electrons are accelerated with the same spectral slope-a reasonable assumption given that DSA depends only on a particle's rigidity-and that synchrotron cooling is negligible at energies corresponding to radio frequencies as discussed in [33], we can conclude that the proton distribution must be similarly steep.

As we discussed above, postcursor physics can reproduce $q \simeq 3$ under the right conditions: specifically, when $v_{\mathrm{sh}}$ is large and the magnetic field is generated by the Bell instability. However, if our intent is to describe a typical radio $\mathrm{SN}$, the models presented above are rather rough approxi- 
mations, since they assume uniform ambient densities and include an injection prescription tuned to observations of Galactic SNRs (i.e., $\xi_{\text {inj }}=3.8$ so that $\xi_{\mathrm{CR}} \approx 0.1$ for a prototypical Galactic remnant). To more accurately approximate the proton distribution of a typical radio SN, we produce a toy-model hydrodynamic evolution that follows an ejecta-dominated radio SN expanding into a circumstellar wind for approximately 10 years. We then use our semi-analytic formalism to self-consistently calculate the corresponding proton spectrum.

More explicitly, we consider an energetic SN $\left(E_{\mathrm{SN}}=10^{52} \mathrm{erg}\right)$ that ejects $M_{\mathrm{ej}}=1 M_{\odot}$ into the circumstellar medium. Since we only model the first 10 years of evolution, the mass swept up by the shock is much smaller than $M_{\mathrm{ej}}$ and we therefore use the approximation in Table 9 of [75] for an ejecta-dominated SNR expanding into a wind: $v_{\mathrm{sh}} \propto t^{-1 / 5}$. For our circumstellar density, we assume a wind profile given by $\rho_{0}=\dot{M} /\left(4 \pi v_{\mathrm{w}}\right)$, where $\dot{M}$ is the mass-loss rate of the progenitor and $v_{\mathrm{w}}$ is the wind velocity. As discussed in [33], we assume typical paramenters for a Wolf-Rayet progenitor: $\dot{M}=10^{-5} M_{\odot} \mathrm{yr}^{-1}$ and $v_{\mathrm{w}}=1000 \mathrm{~km} \mathrm{~s}^{-1}$. We choose an ambient magnetic field that follows our density profile: $B_{0} / \mathrm{G} \simeq 0.01 \sqrt{n_{\mathrm{ISM}} /\left(5000 \mathrm{~cm}^{-3}\right)}$ with normalization chosen such that our magnetic field amplification prescription produces postshock fields consistent with observations [ $B_{2} \sim 0.1-1$ G, e.g., 32]. Finally, since $\xi_{\text {inj }}=3.8$ gives extremely small acceleration efficiencies for our toy model $\left(\xi_{\mathrm{CR}}<0.01\right)$, we reduce $\xi_{\text {inj }}$ slightly to 3.4 . With this adjustment, $\xi_{\mathrm{CR}}$ remains modest $(<0.05)$. The decrease in $\xi_{\text {inj }}$ needed to produce acceleration efficiencies of 5-10\% would yield even steeper spectra.

Our model spectrum is shown in Figure $5 \mathrm{~b}$ and has a slope of $q \simeq 2.99$; note that to make this slope visually apparent, we plot $E^{3} \Phi(E)$. As time passes, each new shell of protons contributes a slightly harder spectrum due to the modest decrease in $v_{\mathrm{sh}}$, which leads to a reduction in magnetic field amplification (for the parameters discussed here, the non-resonant instability dominates). This behavior implies a simple physical explanation for the discrepancy between the very steep spectral slopes of radio SNe and the modestly steep slopes of Galactic SNRs. Namely, as young remnants age and slow down, their postshock magnetic fields decrease, reducing the strength of their postcursors and flattening their spectra.

Note that our model predicts CR spectra to be steep even at high energies, while the classical concave-spectra explanation [e.g., 40, 41, 73] returns rather flat spectra at $\mathrm{TeV}$ energies; therefore, $\mathrm{X}$-ray and, possibly, $\gamma$-ray observations may be able to distinguish between models.

\section{Conclusion}

In summary, we use a semi-analytic model of NLDSA to quantify the CR spectral steepening in SNRs that arises from the presence of a postcursor, i.e., a region behind a shock in which magnetic fluctuations drift away from the shock at the local Alfvén speed with respect to the background fluid. Since CRs isotropize with these fluctuations, they too experience a net drift, leading to a removal of CR energy from the system and thus a steepening of their spectra relative to the standard DSA prediction $\left(\Phi(E) \propto E^{-2}\right)$. The formation of a postcursor has been validated with kinetic simulations $[27,48]$ and provides a natural explanation for the steep CR spectra inferred from observations of SNRs [e.g., 5, 45, 66] and Galactic CRs, once corrected for propagation [e.g., 2, 42].

Because magnetic fluctuations drift with the local Alfvén speed, it is important that we include a prescription for magnetic field amplification that is not only theoretically motivated, but consistent 
with observations. In our model, we implement a self-consistent prescription that incorporates the saturation points of both the resonant [3] and non-resonant $[9,78]$ streaming instabilities. This model yields magnetic fields that are consistent with those inferred from X-ray observations of young SNRs [25, 63, 76, 77] and reproduces the observed relationship between shock velocity and downstream Alfvén speed reported in Zeng et al. [79].

With this prescription for magnetic field amplification [also see 35], our model produces modestly steep spectra $\propto E^{-2.34}$ for a Tycho-like SNR after $400 \mathrm{yr}: n_{\mathrm{ISM}}=1 \mathrm{~cm}^{-3}, B_{0}=3 \mu \mathrm{G}$, $E_{\mathrm{SN}}=10^{51} \mathrm{erg}$, and $M_{\mathrm{ej}}=1 M_{\odot}$. We also confirm that the postcursor is the dominant source of this steepening; neglecting the effect of the precursor still yields spectra $\propto E^{-2.30}$.

As SNRs age and slow down, we find that this steepening diminishes, yielding a power-law slope, $q \simeq 2.23$ for our prototypical SNR after $10^{5} \mathrm{yr}$. Given observational constraints on the slope of the CR diffusion coefficient, this slope is consistent with that needed to reproduce the spectrum of Galactic CRs observed at Earth [e.g., 42, 43].

More generally, for large $v_{\mathrm{sh}}$, the nonresonant instability dominates magnetic field amplification such that the magnetic pressure scales as $v_{\mathrm{sh}} P_{\mathrm{CR}}$. As a result, the downstream Alfvén speed and thus the steepening due to the postcursor diminish as the SNR slows. This dependence largely disappears at lower $v_{\mathrm{sh}}$, when the resonant instability dominates. The location of this transition depends on the ambient density and magnetic field.

The relationship between $v_{\mathrm{sh}}$, magnetic field amplification, and $q$ that arises from postcursor physics provides a theoretically-motivated explanation for the modestly steep spectra of Galactic SNRs $\left(\propto E^{-2.2}\right)$, the very steep spectra of radio $\mathrm{SNe}\left(\propto E^{-3}\right)$, and the connection between them. More specifically, we use our formalism to model both source classes and find that we are able to produce spectra in good agreement with observations.

Our work represents the first generalization of postcursor physics to a wide range of SNR shocks, as well as the first self-consistent quantification of the spectral steepening that arises. The good agreement between our modeled spectra and those inferred from the nonthermal emission of real SNRs implies that the presence of a postcursor may resolve the tension between DSA predictions and observations.

\section{Acknowledgements}

This research was partially supported by a Eugene and Niesje Parker Graduate Student Fellowship, NASA (grants NNX17AG30G and 80NSSC18K1726) and the NSF (grants AST-1909778, PHY-1748958, and PHY-2010240).

\section{References}

[1] Acero et al., F. 2016, , 224, 8, doi: 10.3847/0067-0049/224/1/8

[2] Aguilar et al. [AMS Collaboration], M. 2018, Phys. Rev. Lett., 120, 021101, doi: 10.1103/ PhysRevLett. 120.021101

[3] Amato, E., \& Blasi, P. 2006, MNRAS, 371, 1251, doi: 10.1111/j .1365-2966. 2006.10739. $\mathrm{X}$ 
[4] -. 2009, MNRAS, 392, 1591, doi: 10.1111/j .1365-2966.2008.14200.x

[5] Archambault, S., Archer, A., Benbow, W., et al. 2017, , 836, 23, doi: 10.3847/1538-4357/ $836 / 1 / 23$

[6] Axford, W. I., Leer, E., \& Skadron, G. 1977, in International Cosmic Ray Conference, Vol. 2, Acceleration of Cosmic Rays at Shock Fronts, 273-+. http://adsabs.harvard.edu/abs/ 1977ICRC . . . .2. 273A

[7] Bamba, A., Yamazaki, R., Yoshida, T., Terasawa, T., \& Koyama, K. 2005, ApJ, 621, 793, doi: $10.1086 / 427620$

[8] Bell, A. R. 1978, MNRAS, 182, 147. http://adsabs.harvard.edu/cgi-bin/nph-bib_ query?bibcode=1978MNRAS . 182 . .147B\&db_key=AST

[9] —. 2004, MNRAS, 353, 550, doi: 10.1111/j.1365-2966.2004.08097.x

[10] Bell, A. R., Schure, K. M., \& Reville, B. 2011, , 418, 1208, doi: 10.1111/j . 1365-2966. 2011.19571.x

[11] Berezhko, E. G., \& Ellison, D. C. 1999, ApJ, 526, 385, doi: 10. 1086/307993

[12] Berezhko, E. G., \& Völk, H. J. 1997, APh, 7, 183, doi: 10 . 1016/S0927-6505 (97) 00016-9

[13] —. 2007, , 661, L175, doi: 10.1086/518737

[14] Blandford, R., \& Eichler, D. 1987, , 154, 1, doi: 10.1016/0370-1573(87)90134-7

[15] Blandford, R. D., \& Ostriker, J. P. 1978, ApJL, 221, L29, doi: 10.1086/182658

[16] Blasi, P., \& Amato, E. 2012, , 1, 10, doi: 10.1088/1475-7516/2012/01/010

[17] —. 2012, , 1, 11, doi: $10.1088 / 1475-7516 / 2012 / 01 / 011$

[18] Blasi, P., Amato, E., \& Caprioli, D. 2007, , 375, 1471, doi: 10.1111/j . 1365-2966. 2006. $11412 . \mathrm{x}$

[19] Blasi, P., Amato, E., \& D’Angelo, M. 2015, Physical Review Letters, 115, 121101, doi: 10. 1103/PhysRevLett. 115.121101

[20] Bykov, A. M., Brandenburg, A., Malkov, M. A., \& Osipov, S. M. 2013, , doi: 10.1007/ s11214-013-9988-3

[21] Caprioli, D. 2011, , 5, 26, doi: 10.1088/1475-7516/2011/05/026

[22] —. 2012, , 7, 38, doi: $10.1088 / 1475-7516 / 2012 / 07 / 038$

[23] Caprioli, D., Amato, E., \& Blasi, P. 2010, APh, 33, 160, doi: 10.1016/j . astropartphys. 2010.01 .002

[24] —. 2010, APh, 33, 307, doi: 10.1016/j .astropartphys.2010.03.001 
[25] Caprioli, D., Blasi, P., Amato, E., \& Vietri, M. 2008, ApJ Lett, 679, L139, doi: 10.1086/ 589505

[26] -. 2009, MNRAS, 395, 895, doi: 10.1111/j.1365-2966.2009.14570.x

[27] Caprioli, D., Haggerty, C. C., \& Blasi, P. 2020, , 905, 2, doi: 10.3847/1538-4357/abbe05

[28] Caprioli, D., Pop, A., \& Spitkovsky, A. 2015, ApJ Letters, 798, 28. https://arxiv.org/ abs/1409. 8291

[29] Caprioli, D., \& Spitkovsky, A. 2014, , 794, 46, doi: 10 . 1088/0004-637X/794/1/46

[30] —. 2014, , 794, 47, doi: 10.1088/0004-637X/794/1/47

[31] Cardillo, M., Amato, E., \& Blasi, P. 2016, , 595, A58, doi: 10 . 1051/0004-6361/201628669

[32] Chevalier, R. A. 1998, The Astrophysical Journal, 499, 810, doi: 10.1086/305676

[33] Chevalier, R. A., \& Fransson, C. 2006, , 651, 381, doi: 10.1086/507606

[34] —. 2017, Thermal and Non-thermal Emission from Circumstellar Interaction, ed. A. W. Alsabti \& P. Murdin (Cham: Springer International Publishing), 875-937, doi: 10.1007/ 978-3-319-21846-5_34

[35] Cristofari, P., Blasi, P., \& Caprioli, D. 2021, , 650, A62, doi: 10.1051/0004-6361/ 202140448

[36] Diesing, R., \& Caprioli, D. 2018, Physical Review Letters, 121, 091101, doi: 10.1103/ PhysRevLett. 121.091101

[37] Diesing, R., \& Caprioli, D. 2019, Physical Review Letters, 123, 071101, doi: 10.1103/ PhysRevLett. 123.071101

[38] —. 2021, arXiv e-prints, arXiv:2107.08520. https://arxiv .org/abs/2107.08520

[39] Ellison, D. C., Baring, M. G., \& Jones, F. C. 1996, Ap. J., 473, 1029, doi: 10. 1086/178213

[40] Ellison, D. C., Berezhko, E. G., \& Baring, M. G. 2000, , 540, 292, doi: 10 . 1086/309324

[41] Ellison, D. C., \& Reynolds, S. P. 1991, , 382, 242, doi: 10.1086/170712

[42] Evoli, C., Aloisio, R., \& Blasi, P. 2019, , 99, 103023, doi: 10.1103/PhysRevD . 99. 103023

[43] Evoli, C., Morlino, G., Blasi, P., \& Aloisio, R. 2019, arXiv e-prints, arXiv:1910.04113. https://arxiv.org/abs/1910.04113

[44] Fermi, E. 1954, Ap. J., 119, 1, doi: 10.1086/145789

[45] Giordano, F., Naumann-Godo, M., Ballet, J., et al. 2012, ApJ1, 744, L2, doi: 10.1088/ 2041-8205/744/1/L2 
[46] Green, D. A. 2019, Journal of Astrophysics and Astronomy, 40, 36, doi: 10.1007/ s12036-019-9601-6

[47] H. E. S. S. Collaboration, Abdalla, H., Abramowski, A., et al. 2018, , 612, A6, doi: 10 . 1051/ 0004-6361/201629790

[48] Haggerty, C. C., \& Caprioli, D. 2020, , 905, 1, doi: 10.3847/1538-4357/abbe06

[49] Hillas, A. M. 2005, Journal of Physics G Nuclear Physics, 31, 95, doi: 10 . 1088/0954-3899/ $31 / 5 /$ R02

[50] Jones, F. C., \& Ellison, D. C. 1991, Space Science Reviews, 58, 259. http://adsabs. harvard.edu/abs/1991SSRv ...58..259J

[51] Kamble, A., Margutti, R., Soderberg, A. M., et al. 2016, The Astrophysical Journal, 818, 111, doi: $10.3847 / 0004-637 x / 818 / 2 / 111$

[52] Kang, H., Jones, T. W., \& Edmon, P. P. 2013, , 777, 25, doi: 10.1088/0004-637X/777/1/25

[53] Kang, H., \& Ryu, D. 2018, , 856, 33, doi: 10 .3847/1538-4357/aab1f2

[54] Krymskii, G. F. 1977, Akademiia Nauk SSSR Doklady, 234, 1306. http://adsabs. harvard. edu/cgi-bin/nph-bib_query?bibcode=1977DoSSR. 234R1306K\&db_key=AST

[55] Kulsrud, R., \& Pearce, W. 1968, The Astronomical Journal Supplement, 73, 22

[56] Lagage, P. O., \& Cesarsky, C. J. 1983a, A\&A, 118, 223. http://adsabs.harvard.edu/ abs/1983A26A...118. .223L

[57] Lee, S.-H., Slane, P. O., Ellison, D. C., Nagataki, S., \& Patnaude, D. J. 2013, The Astrophysical Journal, 767, 20, doi: $10.1088 / 0004-637 x / 767 / 1 / 20$

[58] Malkov, M. A., \& O’C. Drury, L. 2001, Rep. Prog. Phys., 64, 429. http://adsabs. harvard.edu/abs/2001RPPh...64..429M

[59] Morlino, G., Amato, E., Blasi, P., \& Caprioli, D. 2010, , 405, L21, doi: 10.1111/j . $1745-3933.2010 .00851 . x$

[60] Morlino, G., \& Caprioli, D. 2012, A\&A, 538, A81, doi: 10.1051/0004-6361/201117855

[61] O’C. Drury, L. 1983, Reports of Progress in Physics, 46, 973. http: //adsabs . harvard. edu/abs/1983RPPh...46. .973D

[62] O’C. Drury, L., \& Völk, H. J. 1981, Ap. J., 248, 344, doi: $10.1086 / 159159$

[63] Parizot et al., E. 2006, A\&A, 453, 387, doi: 10.1051/0004-6361:20064985

[64] Ptuskin, V., Zirakashvili, V., \& Seo, E.-S. 2010, , 718, 31, doi: 10 . 1088/0004-637X/718/ $1 / 31$

[65] Ressler et al., S. M. 2014, , 790, 85, doi: 10.1088/0004-637X/790/2/85 
[66] Saha, L., Ergin, T., Majumdar, P., Bozkurt, M., \& Ercan, E. N. 2014, , 563, A88, doi: 10. $1051 / 0004-6361 / 201323218$

[67] Skilling, J. 1975, MNRAS, 172, 557. http://adsabs.harvard.edu/abs/1975MNRAS. $172 . .557 \mathrm{~S}$

[68] —. 1975b, MNRAS, 173, 245. http://adsabs.harvard.edu/abs/1975MNRAS.173. $.245 \mathrm{~S}$

[69] -. 1975c, MNRAS, 173, 255. http://adsabs.harvard.edu/abs/1975MNRAS.173. $.255 \mathrm{~S}$

[70] Slane, P., Lee, S.-H., Ellison, D. C., et al. 2014, , 783, 33, doi: 10.1088/0004-637X/783/ $1 / 33$

[71] Soderberg, A. M., Brunthaler, A., Nakar, E., Chevalier, R. A., \& Bietenholz, M. F. 2010, The Astrophysical Journal, 725, 922, doi: 10 . 1088/0004-637x/725/1/922

[72] Soderberg, A. M., Margutti, R., Zauderer, B. A., et al. 2012, The Astrophysical Journal, 752, 78, doi: $10.1088 / 0004-637 x / 752 / 2 / 78$

[73] Tatischeff, V. 2009, , 499, 191, doi: 10.1051/0004-6361/200811511

[74] Tran, A., Williams, B. J., Petre, R., Ressler, S. M., \& Reynolds, S. P. 2015, , 812, 101, doi: $10.1088 / 0004-637 \mathrm{X} / 812 / 2 / 101$

[75] Truelove, J. K., \& Mc Kee, C. F. 1999, ApJ Supplement Series, 120, 299, doi: 10.1086/ 313176

[76] Vink, J., \& Laming, J. M. 2003, Ap. J., 584, 758, doi: 10 . 1086/345832

[77] Völk, H. J., Berezhko, E. G., \& Ksenofontov, L. T. 2005, A\&A, 433, 229, doi: 10.1051/ 0004-6361:20042015

[78] Zacharegkas, G., Caprioli, D., \& Haggerty, C. 2019, in International Cosmic Ray Conference, Vol. 36, 36th International Cosmic Ray Conference (ICRC2019), 483. https://arxiv.org/ abs/1909.06481

[79] Zeng, H., Xin, Y., \& Liu, S. 2019, , 874, 50, doi: 10.3847/1538-4357/aaf392

[80] Zirakashvili, V. N., \& Ptuskin, V. S. 2008, astro-ph/0807.2754. https://arxiv .org/abs/ 0807.2754

[81] Zweibel, E. G. 1979, in American Institute of Physics Conference Series, Vol. 56, Particle Acceleration Mechanisms in Astrophysics, ed. J. Arons, C. McKee, \& C. Max, 319-328, doi: 10. 1063/1.32090 